Research Paper

\title{
Vitamin D inhibits the proliferation of Oral Squamous Cell Carcinoma by suppressing IncRNA LUCAT1 through the MAPK pathway
}

Tingting Jin ${ }^{1 *}$, Ying Guo ${ }^{2 *}$, Zixian Huang1, Qianyu Zhang1, Zhuoshan Huang3, Yin Zhang4\#® , and Zhiquan Huang1\#®

1. Department of Oral and Maxillofacial Surgery, Sun Yat-sen Memorial Hospital, Sun Yat-sen University, 107 Yan-jiang Road, Guangzhou, Guangdong Province, China, 510120.

2. Department of Endocrinology, Sun Yat-sen Memorial Hospital, Sun Yat-sen University, 107 Yan-jiang Road, Guangzhou, Guangdong Province, China, 510120.

3. Department of Stomatology, Sun Yat-sen Memorial Hospital,Sun Yat-sen University, 107 Yan-jiang Road, Guangzhou, Guangdong Province, China, 510120.

4. Key Laboratory of Malignant Tumor Epigenetics and Gene Regulation, Research Center of Medicine, Sun Yat-sen Memorial Hospital, Sun Yat-sen University, Guangzhou 510120, P. R. China.

* Tingting Jin and Ying Guo shared the first authorship.

\# Yin Zhang and Zhiquan Huang shared the corresponding author.

$\square$ Corresponding authors: Professor Dr. Yin Zhang, E-mail: zhangy525@mail.sysu.edu.cn. And/Or Dr. Zhi-quan Huang, DDS, MD, E-mail: zhiquanhuang1978@126.com; Tel.: +86 02081332429.

( ) The author(s). This is an open access article distributed under the terms of the Creative Commons Attribution License (https://creativecommons.org/licenses/by/4.0/). See http:/ /ivyspring.com/terms for full terms and conditions.

Received: 2020.02.27; Accepted: 2020.08.02; Published: 2020.08.15

\begin{abstract}
Background: Oral squamous cell carcinoma (OSCC) is the most common type of oral cancer worldwide. Recent studies have suggested that vitamin $D(V i t D)$ is associated with a reduced risk of many chronic illnesses, including cancer. However, the role of vitamin D in OSCC has rarely been reported.

Materials and Methods: The effect of vitamin $D$ and control treatment were examined by cell clone formation assay. Using RNA-seq, we globally identified VitD-regulated long noncoding RNAs (IncRNAs). The expression of LUCATI in OSCC tissues and cell lines was examined by GRT-PCR. The correlation between LUCATI expression level and clinicopathological characteristics was analyzed. The biological roles of LUCAT1 in OSCC cell proliferation was determined by CCK8 and cell colony formation. The role of LUCAT1 in OSCC growth was further confirmed by mouse xenograft tumor model. Combined with the literature, the mechanism of action of LUICATI was verified by western blot.

Results: In this study, we observed that VitD inhibited tumour cell growth in OSCC. We found that IncRNA LUCATI was downregulated by VitD and served as an important mediator of VitD in inhibiting OSCC cell proliferation. Moreover, we observed that the expression of LUCATI was significantly upregulated in OSCC tissues compared to non-tumour tissues. We further demonstrated that LUCATI promoted the proliferation of oral cancer cells by enhancing the activation of the mitogen protein kinase (MAPK) signalling pathway.

Conclusion: In summary, our results show that VitD inhibited the growth of OSCC cells through the LUCATI-MAPK signalling pathway. Our study suggested that VitD could suppress the progression of oral cancer, and LUCATI may be a potential tumour marker for the diagnosis and prognosis of OSCC.
\end{abstract}

Key words: Vitamin D; Oral squamous cell carcinoma; LUCAT1; Cell proliferation

\section{Introduction}

According to the latest statistics by the American Cancer Society, oral squamous cell carcinoma (OSCC) and oropharynx carcinoma are the ninth most prevalent cancers worldwide [1]. Despite advances in surgical techniques and postoperative radiotherapy, the overall 5-year survival of OSCC patients has not 
significantly improved in the last 50 years. The 5 -year survival rate for OSCC patients is still only approximately $40 \%-50 \%$ [2.3]. The lack of suitable early detection targets and new molecular-targeted antitumour treatments remains a major challenge for OSCC therapy [4]. Moreover, the molecular mechanism underlying OSCC development is still unclear. Few targetable genomic lesions have been identified and no molecular therapy is available [5]. Therefore, we sought to explore the carcinogenic mechanism of OSCC to find new targets for OSCC treatment.

Vitamin D (VitD) is a derivative of fat-soluble steroids (also known as 1,25-dihydroxy-vitamin D3 $(1,25(\mathrm{OH}) 2 \mathrm{D} 3))$ that can be divided into vitamin D2 and vitamin D3. The body acquires VitD mainly through ultraviolet radiation and only a small portion is obtained from food. One of the first described classic effects of VitD is its ability to regulate calcium and phosphorus metabolism and promote bone mineralisation. Increasing epidemiological data suggest an important role of VitD signalling in cancer development and progression, and experimental studies demonstrate that the active VitD metabolite 1a,25-dihydroxy-VitD $3\left(1,25 \mathrm{D}_{3}\right)$, has broad antitumour activity [6]. 1,25-Dihydroxy-VitD $\mathrm{D}_{3}$ exerts a suppressive effect on kidney cancer cells via upregulation of FOXO3 [7]. VitD analogues suppress IGF-I signalling and promote apoptosis in breast cancer cells [8]. Our previous research found that VitD can inhibit the progression of adenoid cystic carcinoma and oesophageal squamous cell carcinoma [9, 10]. Recently, we further found that VitD promoted the cisplatin sensitivity of oral squamous cell carcinoma [11]. All these results indicate that VitD may play an important role in the prevention and treatment of tumours.

Long noncoding RNAs (lncRNAs) are defined as transcripts with a length of at least $200 \mathrm{nt}$ that are unlikely to be translated into proteins. It is reported that only $2 \%$ of human genome encodes for proteins [12], the number of lncRNAs is more than twice as many as protein-coding genes [13]. LncRNAs have been reported to play crucial roles in multiple biological processes, including tumorigenesis and progression. In recent years, the important role of lncRNAs in tongue cancer has been studied. Dai et al. found high expression of HOTAIR in TSCC. They noted that HOTAIR participates in the regulation of proliferation and apoptosis of TSCC cells [14]. Liang et al. found that lncRNA MALAT1 expression was upregulated in tongue tissues and correlated with cervical lymph node metastasis [15]. However, there is no research on whether VitD has antitumour effects through lncRNAs in TSCC, and the underlying mechanisms of Vitamin D's anti-tumor effect at the lncRNA layer is still largely unknown.

Here, we report a genome-wide study on vitDregulated lncRNAs through an integrated analysis of strand-specific RNA-seq and TCGA clinical data. Moreover, we observed that LUCAT1 is an important vitD-regulated lncRNA and that transiently or stably silenced LUCAT1 significantly inhibited OSCC proliferation. These findings reveal that VitD can inhibit the proliferation of OSCC by regulating the LUCAT1/MAPK signalling pathway. Our study provides insights for the links between lncRNAs and Vitamin D effects.

\section{Materials and Methods}

\section{Antibodies}

Mouse monoclonal anti-FLAG (\#F3165) was purchased from Sigma. Anti-phospho-p44/42 MAPK (Erk1/2) (Thr202/Tyr204) antibody \#9101, antip44/42 MAPK (Erk1/2) (137F5) antibody (\#4695), anti-rabbit IgG, HRP-linked antibody 7074, and antiGAPDH antibody (\#D16H11) were purchased from Cell Signaling Technology. Antigen Ki-67 (YM6189) was purchased from Immunoway.

\section{Reagents}

The VitD metabolite 1,25D3 (Sigma, USA) was dissolved at a concentration of $400 \mu \mathrm{M}$ in anhydrous alcohol (AA) for preservation. Immediately prior to use, the stock was diluted to a final concentration of $30 \mathrm{~nm}$ in culture medium. Si-RNA LUCAT1 was purchased from GenePharma (Suzhou, China). Other chemicals were purchased from Sigma. All of the culture media (DMEM for CAL-27, DMEM-F12 for SCC-9) and foetal bovine serum (FBS) were purchased from Bioind.

\section{OSCC sample collection and patient follow-up}

To address the research aim, patients seen at the Department of Oral and Maxillofacial Surgery, Sun Yat-sen Memorial Hospital between 2017 and 2018 for the treatment of OSCC were recruited. Inclusion criteria included a pathological diagnosis of OSCC and willingness to participate in the subsequent follow-up. Patients were excluded from the study if they were diagnosed with multiple cancers or other severe diseases. Data on the characteristics of the OSCC patients, including age, gender, tumour differentiation, lymphatic metastasis and clinical stage, were collected. All patients had an appointment at least every season. In addition, patient tumour samples and adjacent noncancerous (ANC) samples were collected. The ANC tissue refers to an area at least $2 \mathrm{~cm}$ from the tumour lesion that represents the resection border and is pathologically confirmed as 
noncancerous tissue.

\section{Cell culture and transfection}

The human oral squamous cell carcinoma cell lines CAL27 and SCC9 and the lentivirus vector packaging cell line HEK293T were obtained from the American Type Culture Collection (ATCC). All OSCC cell lines were routinely cultured in DMEM and DMEM-F12 medium supplemented with $10 \%$ FBS in a $37^{\circ} \mathrm{C}$ humidified incubator containing $5 \% \mathrm{CO} 2$. All the cell lines were validated by short tandem repeat profiling analysis and were free of mycoplasma contamination.

Transient transfection of OSCC cells was performed using Lipofectamine 3000 (Invitrogen) reagent according to the manufacturer's instructions. For transient transfections using small interfering RNAs (siRNAs), siRNAs targeting LUCAT1 were synthesised by GenePharma (Suzhou, China). The transfection was performed with Lipofectamine 3000 reagent (Invitrogen, Carlsbad, USA) according to the manufacturer's protocol. The siRNA sequences are shown in Table 1.

For stable expression, short hairpin RNA targeting LUCAT1 (shLUCAT1\#1 and shLUCAT1\#1) and the corresponding control scramble were designed and synthesised by Genepharma (Shanghai, China). Subsequently, RT-qPCR was performed to confirm the correct expression of the stable cell lines. We selected shLUCAT1\#2 due to its stronger knockdown effect for the subsequent experiment.

Table 1. The siRNA sequences

\begin{tabular}{lll}
\hline Gene & Sense $\left(5^{\prime}-3^{\prime}\right)$ & Antisense $\left(5^{\prime}-3^{\prime}\right)$ \\
\hline LUCAT1\#1 & CCCAUCAGAAGAUGUCAGA & UUAUCUUCUGACAUCUUCU \\
& AGAUAA & GAUGGG \\
LUCAT1\#2 & CAAGCUCUUGCAGUCAACA & AGUUCUUGUUGACUGCAAG \\
& AGAACU & AGCUUG \\
\hline
\end{tabular}

\section{Immunohistochemistry (IHC)}

Immunohistochemical staining was performed according to standard protocols. After deparaffinisation, antigen retrieval was conducted using $10 \mathrm{mM}$ sodium citrate buffer $(\mathrm{pH} 8.0$ ) in a pressure cooker at full power for $5 \mathrm{~min}$. Briefly, the tissue sections were blocked sequentially with $3 \% \mathrm{H}_{2} \mathrm{O}_{2}$ and normal serum and then incubated with primary antibodies at $4^{\circ} \mathrm{C}$ overnight. The tissue sections were incubated with a biotinylated secondary antibody and conjugated with a streptavidin-HRP complex (readyto-use SP kit; Zhongshan Co. Beijing, China). Finally, the sections were visualised with 3-3'diaminobenzidine, counterstained with haematoxylin and mounted. The samples were rinsed with phosphatebuffered saline (PBS) between each step.

\section{Western blot}

For protein extraction, the cells were washed twice with cool PBS, harvested by scraping and then lysed in lysis buffer (Beyotime, China). Following centrifugation, the supernatant was collected, and the protein concentration was determined using the BCA Protein Assay Kit (Pierce, USA). For western blotting, cell lysates were electrophoretically separated on an SDS-PAGE gel using a standard protocol. The proteins were then transferred to polyvinylidene fluoride (PVDF) membranes (IPVH00010; Millipore, USA). The membranes were blocked with $5 \%$ non-fat milk in Tris-buffered saline containing $0.1 \%$ Tween-20 (TBST) for $1 \mathrm{~h}$ at room temperature. The blots were then incubated with the antibodies mentioned above at $4^{\circ} \mathrm{C}$ overnight, washed in TBST and then probed with secondary antibody. Western blot analysis was performed using the band intensity measurements of the blots.

\section{RNA extraction, real-time quantitative RT-PCR and RNA sequencing}

Total RNA was extracted using TRIzol reagent (Takara, Japan) according to the manufacturer's instructions and then reverse transcribed into cDNA using PrimeScript ${ }^{\mathrm{TM}}$ RT Master Mix (Takara, Japan) on an ABI 9700 Real-Time PCR system (ABI, USA). The newly synthesised cDNA was then used as a template for the detection of the desired gene. Specifically, $1 \mu \mathrm{l}$ of cDNA was mixed with TB Green ${ }^{\circledR}$ Premix Ex Taq ${ }^{\mathrm{TM}}$ II (Takara, Japan) in a $20-\mu$ l reaction. All of the reactions were run in triplicate using the primers described above. The reaction conditions were as follows: $94^{\circ} \mathrm{C}$ for $2 \mathrm{~min}, 94^{\circ} \mathrm{C}$ for $20 \mathrm{~s}, 58^{\circ} \mathrm{C}$ for $20 \mathrm{~s}$ and $72^{\circ} \mathrm{C}$ for $20 \mathrm{~s}$, for 40 cycles. The relative expression of mRNA was detected using the Roche LightCycler 480 II Real-time PCR machine (Roche, USA). The primer sequences are shown below in Table 2.

Table 2. The primer sequences

\begin{tabular}{lll}
\hline Gene & Forward & Reverse \\
\hline LUCAT1 & GCTCGGATTGCCTTAGACAG TGCCAAGGTCCCATAAGAGT \\
GAPDH & GAGTCAACGGATTTGGTCGT & GACAAGCTTCCCGTTCTCAG
\end{tabular}

\section{Cell proliferation assay}

CAL27 and SCC9 cells were seeded at a density of 1000 cells per well into 96-well plates in triplicate and cultured for 24 to 72 hours after transfection. Before observation, each well was treated with 10 $\mu \mathrm{L} /$ well of cell counting kit-8 solution (Dojindo, Japan) during the last 4 hours of culture. Then, cell viability was determined by measuring the absorbance of the converted dye at $450 \mathrm{~nm}$. For the 
colony formation assay, specific numbers of transfected cells were placed into each well of a six-well plate and cultured in medium containing $10 \%$ FBS for 15 days, replacing the medium every 5 days. Colonies were fixed with methanol and stained with $0.1 \%$ crystal violet (Sigma-Aldrich, St. Louis, MO, USA) in PBS for $15 \mathrm{~min}$. Colony formation was determined by counting the number of stained colonies. For each treatment group, wells were counted in triplicate.

\section{Statistical analyses}

All statistical analyses were conducted using SPSS 22.0 statistical software (IBM, USA). The survival curves were plotted using the Kaplan-Meier method and compared with the log-rank test. Student's $t$ test was used to compare the RT-qPCR results, tumour xenograft results, and cell proliferation between the different groups. Unless otherwise noted, quantitative data are expressed as the mean and standard error of the mean (S.E.M). Statistical significance was determined with a paired
Student's $t$ test. $\quad{ }^{*} P<0.05 ; \quad{ }^{*} P<0.01 ; \quad * * * P<0.001$, compared with the control.

\section{Results}

\section{VitD inhibited cell proliferation in OSCC}

Our previous study demonstrated the role of vitamin D in chemotherapy resistance in OSCC, and we further explored other effects of vitamin $D$ on OSCC. To investigate the effect of LUCAT1 on OSCC cell growth, cell proliferation was measured by colony formation assays. CAL27 and SCC9 cells were pretreated with $30 \mathrm{nM}$ VitD for 3 days, and then a colony formation assay was performed. The results revealed that the colony formation abilities of CAL27 and SCC9 cells pretreated with VitD were decreased (Figure 1A).

\section{LUCATI was the key target in VitD-regulated proliferation of OSCC}

We pretreated CAL27 cells with $30 \mathrm{nM}$ VitD for $72 \mathrm{~h}$. Then, total RNA was extracted for high-
A

\section{CAL 27}
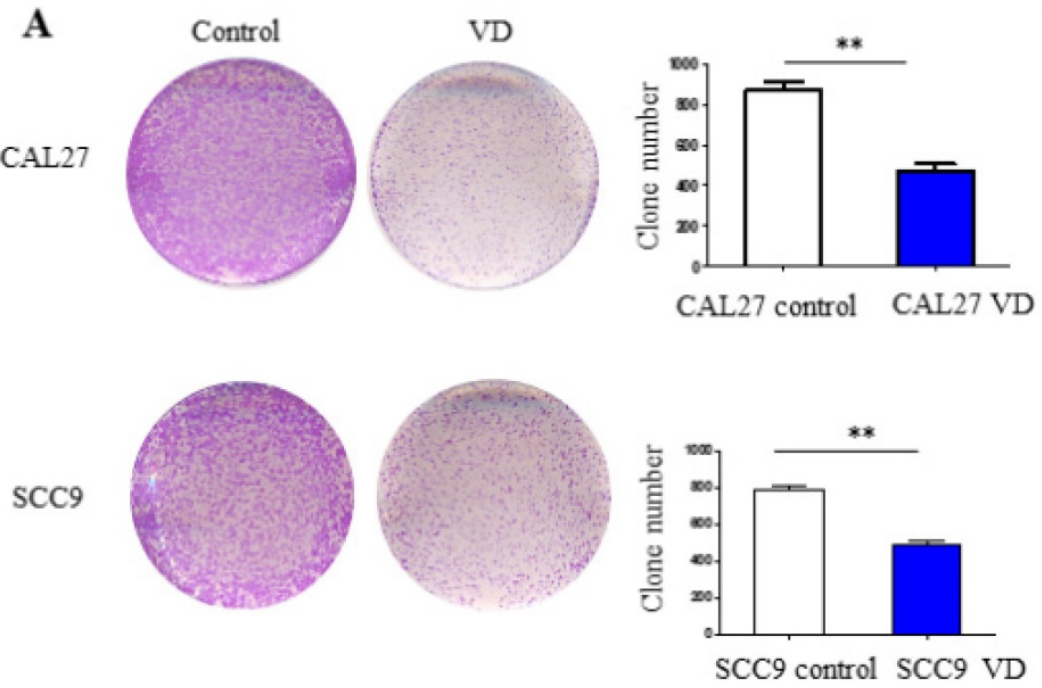

C

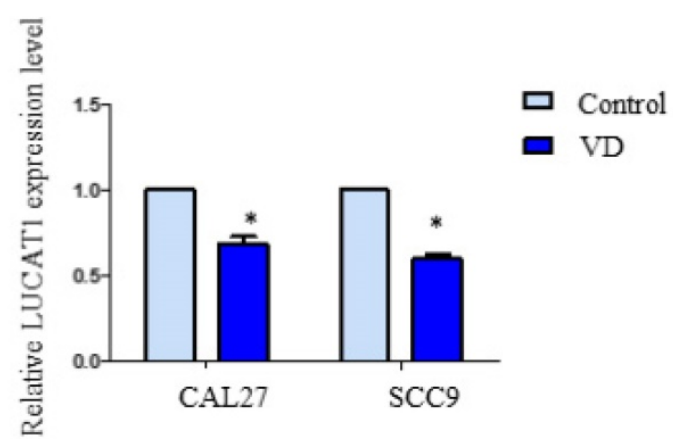

B

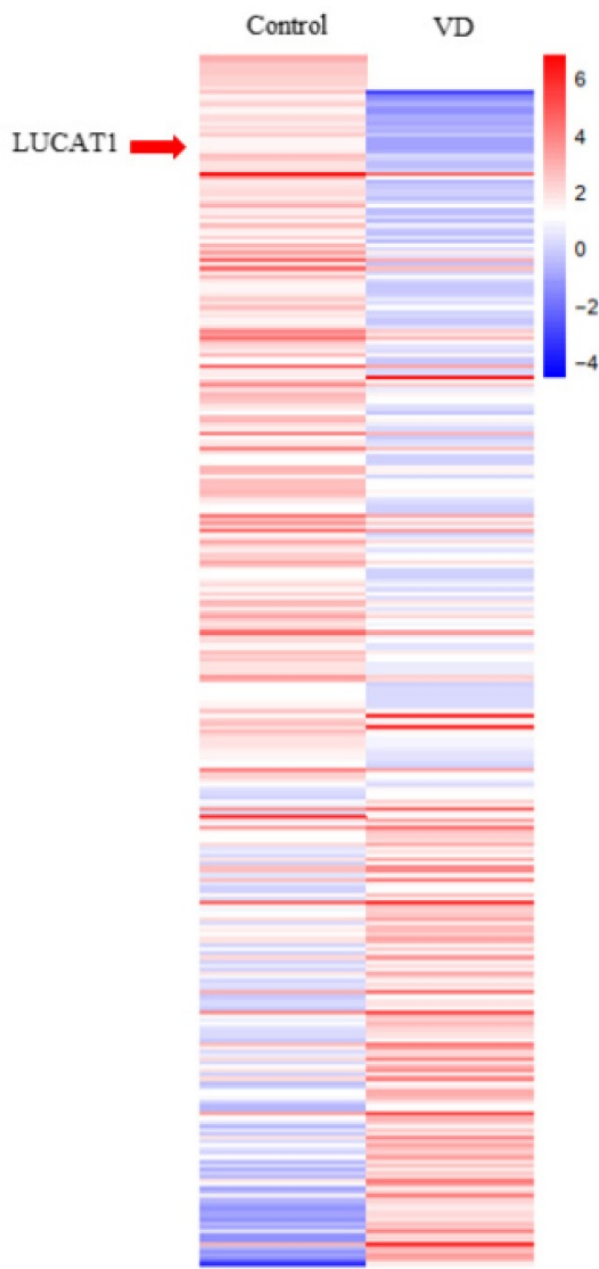

Figure 1. Vitamin D inhibits TSCC cell proliferation and identification of VitD-regulated IncRNAs. A. The colony formation assay results in CAL27 and SCC9 cells treated with vitamin D. B. Heatmap of high-throughput sequencing data in CAL27 cells treated with PBS (control) or vitamin D (Vit D). C. RT-qPCR detection of the expression levels of LUCAT1 with vitamin D treatment in SCC9 and CAL27 cells. Error bars indicate the means \pm S.E.M. $* P<0.05, * * P<0.01$. $* * * P<0.001$. 
throughput sequencing. In addition to the changes in protein-coding genes, we found that many lncRNAs showed significant changes after VitD treatment. We further carried out systematic identification of VitDregulated lncRNAs. We found that 1045 VitDregulated lncRNAs showed significant differential expression between VitD group and control group in OSCC cell (Table S1). LUCAT1 was one of the most significantly changed VitD-regulated lncRNAs (Figure 1B). We verified the results by RT-qPCR, and similar results were obtained for both CAL27 and SCC9 cells (Figure 1C).

\section{LUCATI was highly expressed in OSCC and associated with clinicopathological parameters}

The expression levels of abnormally expressed lncRNAs in human head and neck cancer samples was evaluated using TCGA RNA-seq data. The differentially expressed lncRNAs were screened, and the most significantly dysregulated lncRNAs are shown in the heatmap (Figure 2A). In addition, the expression of LUCAT1 was significantly upregulated in head and neck cancer tissues compared to normal tissues in TCGA datasets (Figure 2B). We further performed RT-qPCR to measure the expression pattern of LUCAT1 in our own cohort of 46 pairs of OSCC tissues and adjacent noncancerous oral tissues, and the results suggested that LUCAT1 was highly expressed in OSCC tissues compared to normal tissues $(p<0.01)$. To explore the clinical significance of LUCAT1 in OSCC, the 46 patients with OSCC were divided into several groups according to their postoperative pathology. As shown in Table 3, there was a statistically significant difference in the expression of LUCAT1 between different tumour sizes. Tumour size was associated with the expression of LUCAT1. However, there was no significant difference between LUCAT1 expression and other clinicopathological factors.
A

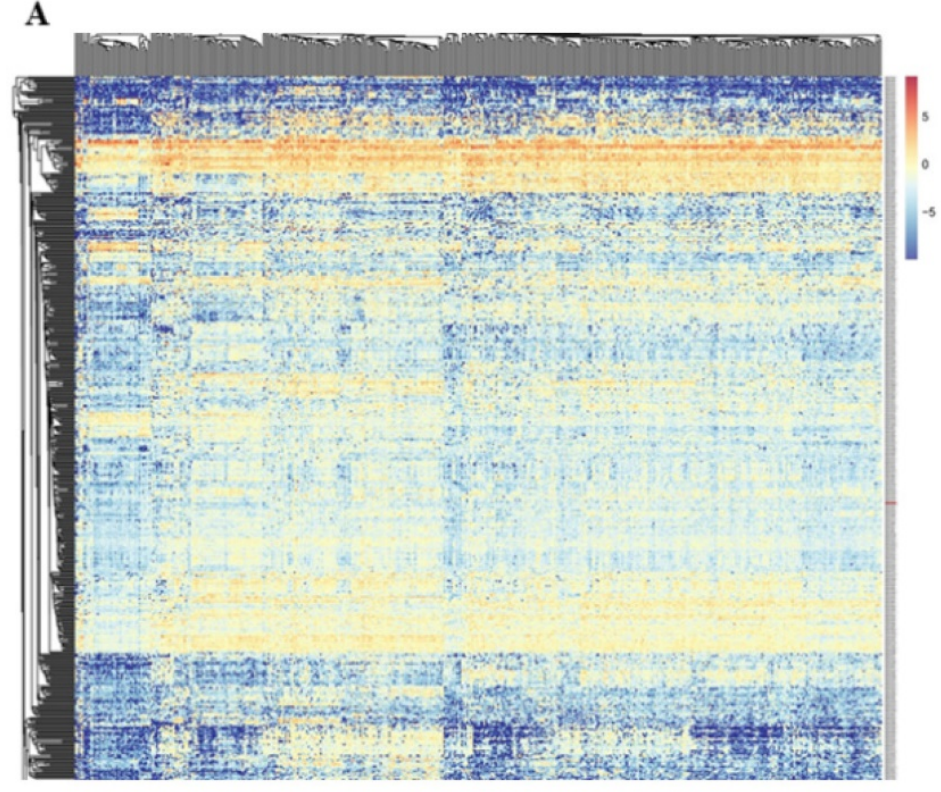

B

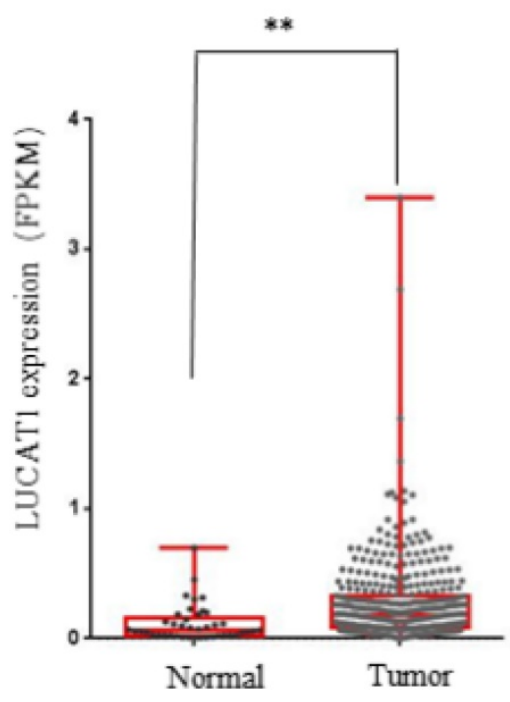

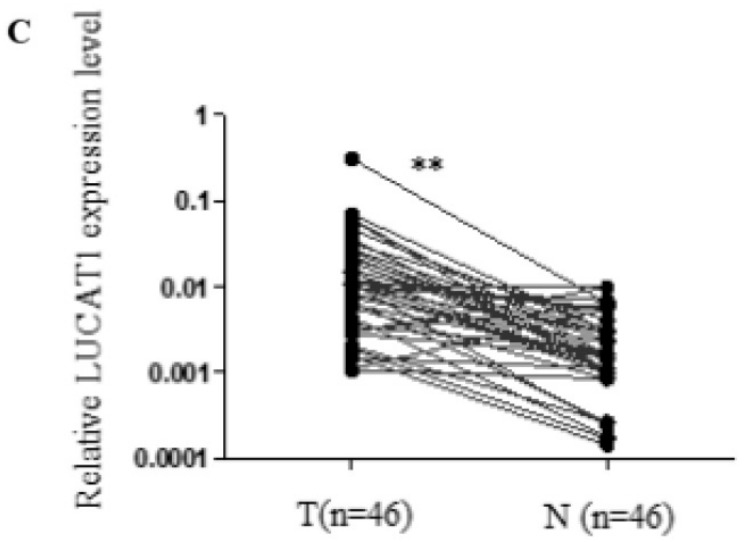

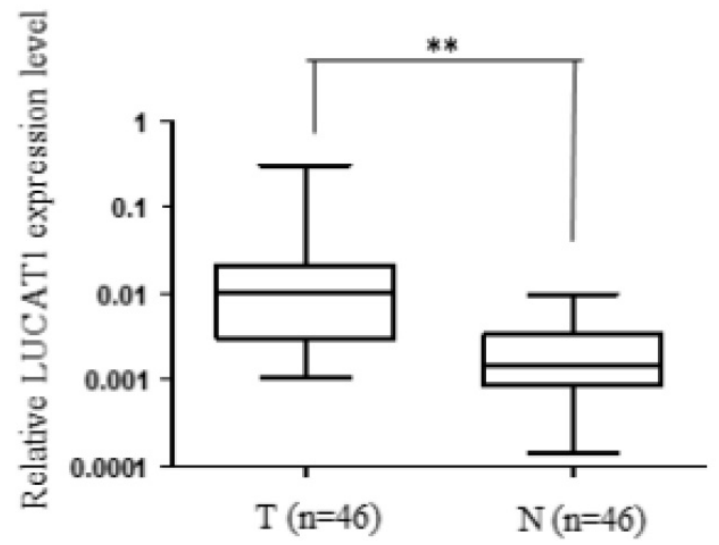

Figure 2. LUCAT1 expression is increased in TSCC and is associated with clinicpathological Parameters. A, B. Analysis of RNA-seq data of LUCAT1 in TCGA. C. LUCAT1 expression in TSCC tissues $(n=46)$ increased significantly compared to that in the corresponding non-tumour tissues $(n=46)$. LUCAT1 expression was detected by RT-PCR and normalised to GAPDH expression. Error bars indicate the means \pm S.E.M. $* P<0.05$, $* * P<0.01$. $* * * P<0.001$. 
A

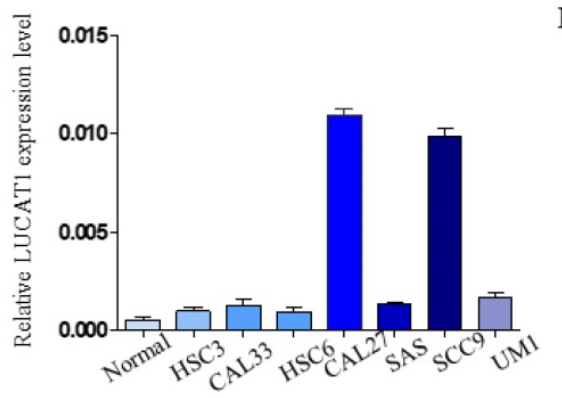

C

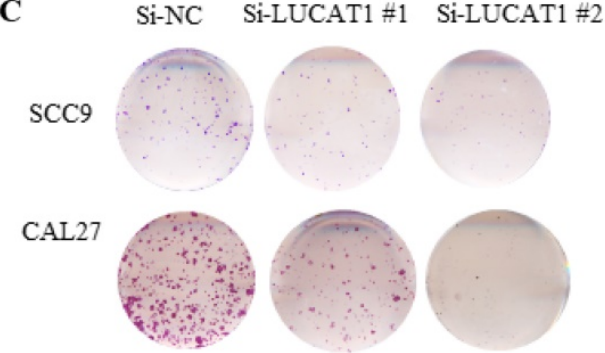

D

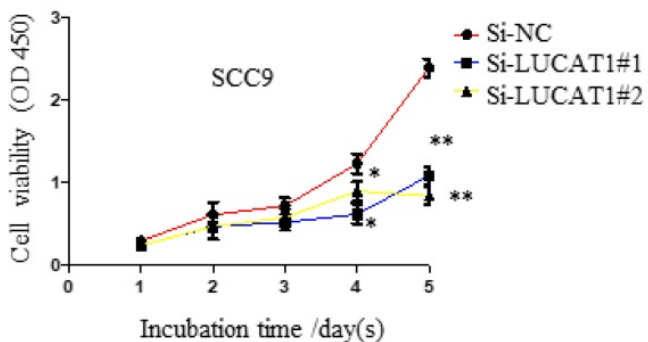

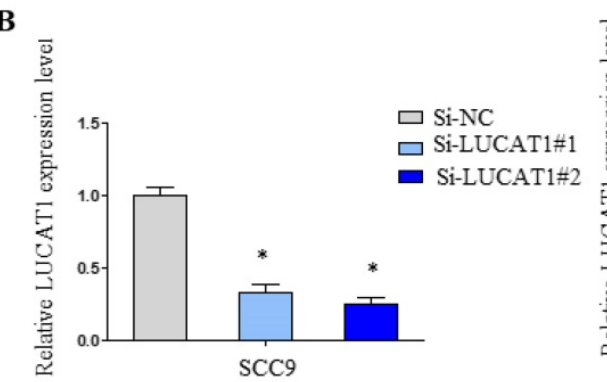
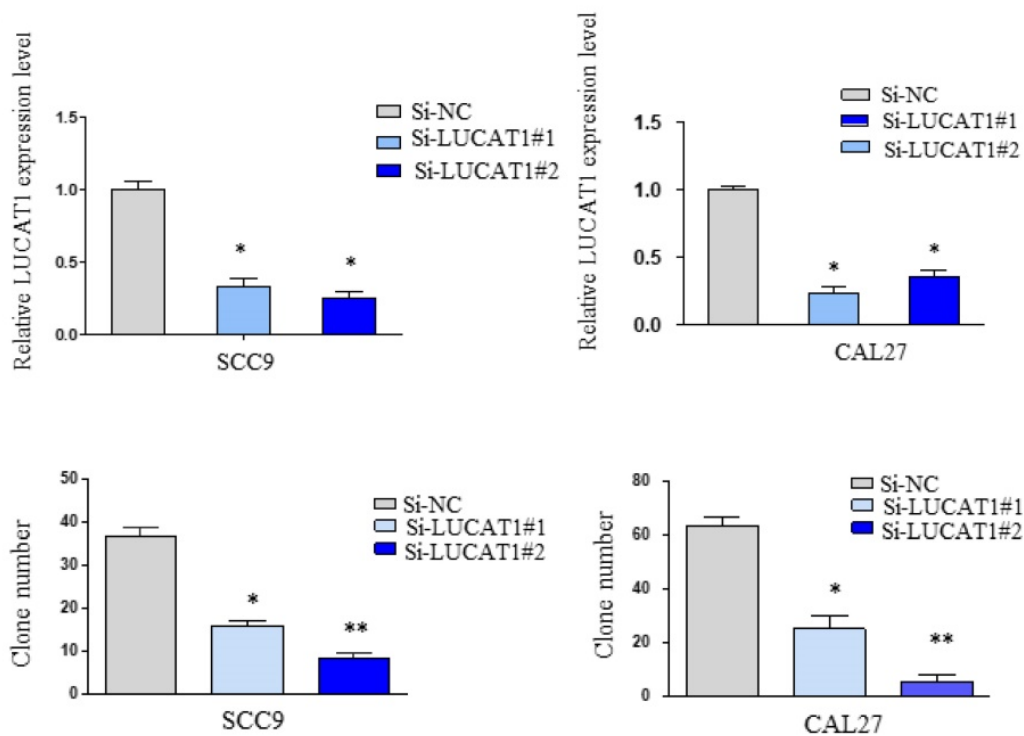

Figure 3. LUCAT1 regulates TSCC cell growth in vitro. A. Relative expression of LUCAT1 in TSCC cell lines. B. At $48 \mathrm{~h}$ after transfection, LUCAT1 expression was analysed by RT-qPCR. C. The results of colony formation in SCC9 and CAL27 cells transfected with siRNA against LUCAT1. D. At $48 \mathrm{~h}$ after transfection, a CCK-8 assay was performed to detect the proliferation of SCC9 and CAL27 cells. Error bars indicate the means \pm S.E.M. $* P<0.05$, $* * P<0.01$. $* * * P<0.001$.

Table 3. Relationship between IncRNA LUCAT1 expression and clinicopathologic parameters in 46 TSCC patients

\begin{tabular}{llll}
\hline Characteristics & Number $(\%)$ & LUCAT1 expression & $P$ value \\
\hline Tumour size & $6(13.04 \%)$ & $0.0017 \pm 0.0019$ & \\
T1 & $16(34.78 \%)$ & $0.0064 \pm 0.0030$ & \\
T2 & $12(26.09 \%)$ & $0.1510 \pm 0.0515$ & \\
T3 & $12(26.09 \%)$ & $0.8810 \pm 0.1055$ & 0.0013 \\
T4 & & & \\
Differentiation & $10(21.74 \%)$ & $0.0122 \pm 0.02125$ & \\
Well & $23(50 \%)$ & $0.0432 \pm 0.08681$ & \\
Moderate & $13(28.26 \%)$ & $0.0180 \pm 0.00994$ & 0.1413 \\
$\begin{array}{l}\text { Poor } \\
\text { Lymphatic metastasis }\end{array}$ & & & \\
Yes & $21(45.65 \%)$ & $0.0821 \pm 0.1034$ & \\
No & $25(54.55 \%)$ & $0.0287 \pm 0.0629$ & 0.0617 \\
\hline
\end{tabular}

\section{Downregulation of LUCATI expression inhibited OSCC cell growth in vitro}

To further understand the potential role of LUCAT1 in OSCC, RT-qPCR analysis was used to detect the expression of LUCAT1 in OSCC cell lines (normal oral mucosa epithelium cells, HSC3, HSC6, CAL27, CAL33, SAS, UM1 and SCC9). As shown in Figure 3A, LUCAT1 had the highest relative expression in CAL27 and SCC9 cell lines. Therefore, both CAL27 and SCC9 cells were chosen for further study. LUCAT1 was knocked down by transfection of LUCAT1 siRNA into CAL27 and SCC9 cells. After 48 $h$ of transfection, RT-qPCR results showed that LUCAT1 expression was markedly reduced (Figure 3B).

To explore the effect of LUCAT1 on OSCC cell growth, cell proliferation was measured by colony formation and CCK-8 assays. The downregulation of LUCAT1 notably suppressed the colony formation abilities of SCC9 and CAL27 cells (Figure 3C). In addition, the growth curves generated by CCK-8 assays indicated that knockdown of LUCAT1 expression remarkably impaired SCC9 and CAL27 cell growth, which was consistent with the colony formation results (Figure 3D).

We used CAL27 shLUCAT1 cells for tumourigenesis in vivo. This analysis also revealed that shLUCAT1\#2 had a higher silencing efficiency than shLUCAT1\#1 (Figure 4A), so we chose shLUCAT1\#2 for further experiments. As shown in Figure 4B, knockdown of LUCAT1 expression markedly inhibited cell proliferation compared to that in the control cells. 
A

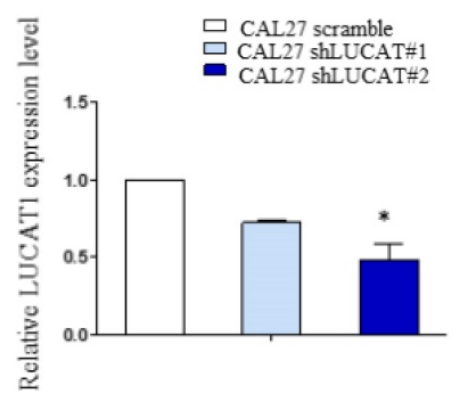

C

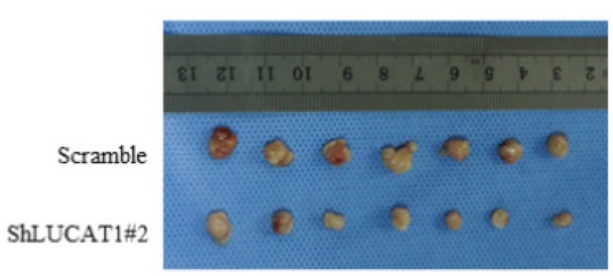

$\mathbf{E}$

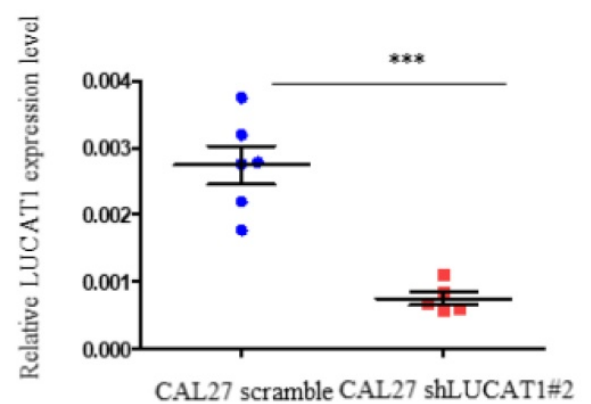

B
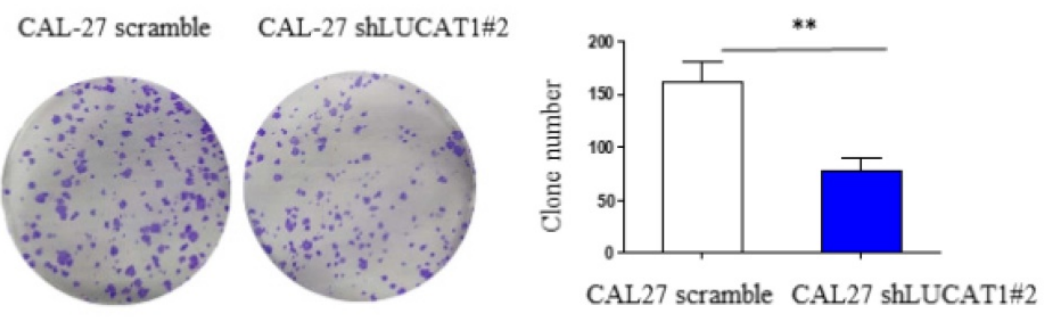

D
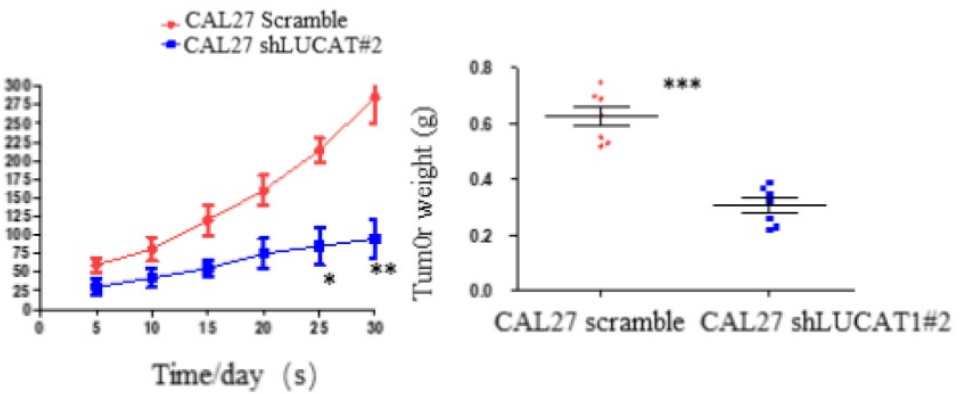

$\mathbf{F}$

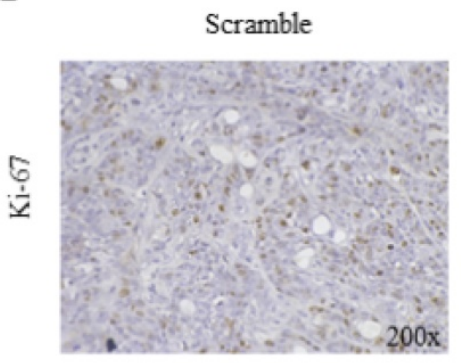

ShLUCAT1 $\# 2$

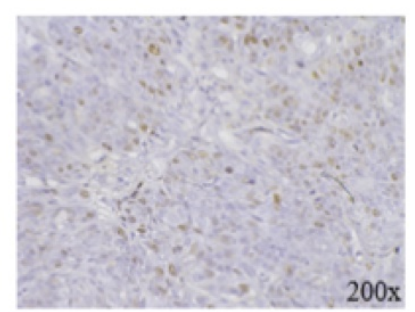

Figure 4. The impact of LUCAT1 on tumourigenesis in vivo. A. RT-PCR detection of the expression levels of LUCAT1 in CAL27 shLUCAT1 cells. B. Colony formation result in CAL27 shLUCAT1 cells. C and D. Scramble and shLUCAT1 were injected into nude mice $(n=7)$. The tumour volumes were calculated every 5 days after injection. The bars indicate SD. E. The tumour weights are shown as the means of tumour weights \pm S.D. F. Histopathology of xenograft tumours. The tumour sections were subjected $\mathrm{IHC}$ staining using an antibody against Ki-67. Error bars indicate the means \pm S.E.M. $* P<0.05, * * P<0.01 . * * * P<0.001$.

\section{Knockdown of LUCAT1 inhibited OSCC cell proliferation in vivo}

To determine the effects of LUCAT1 on tumourigenesis in vivo, we subcutaneously injected CAL27 cells transfected with either shLUCAT1\#2 or scrambleinto the flanks of 5-week-old nude mice. Five days after injection, all mice developed xenograft tumours at the injection site. We measured the xenograft diameters and weighed mice every 5 days and found that tumour growth in the shLUCAT1 group was measurably slower than that in the scramblegroup (Figure 4C). Moreover, the average tumour weight and volume were distinctly lower in the shLUCAT1 group than in the scramble group (Figure 4D). RT-qPCR results showed that LUCAT1 was significantly higher in tumours of the scramble group than the shLUCAT1 group (Figure 4E). Additionally, we found that the xenografts generated by CAL27 shLUCAT1 cells had lower Ki-67 expression than the xenografts generated by CAL27 scramblecells (Figure 4F). As shown in Table 4, the difference is statistically significant. These results provide further evidence that the downregulated expression of LUCAT1 was significantly correlated with the decreased proliferative capacity of OSCC cells in vivo.

Table 4. Statistical analysis of Ki-67 expression in xenografts

\begin{tabular}{llll}
\hline \multirow{2}{*}{ Group } & \multicolumn{2}{l}{ Ki67 expression } & P value \\
\cline { 2 - 4 } & High & Low & \\
\hline Scarmel & 6 & 1 & 0.029 \\
Sh-LUCAT & 1 & 6 & 0.00
\end{tabular}

\section{VitD inhibited the MAPK signalling pathway of OSCC by inhibiting the expression of LUCAT1}

It has been reported that VitD inhibits MAPK signalling pathway activation [16]. The MAPK signalling pathway participates in the physiological functions of various cancer cells in vivo, including proliferation, apoptosis and differentiation [17]. To detect the effect of VitD on the MAPK signalling pathway in OSCC cells, we detected the key proteins 
ERK1/2, p38 and JNK. We found that phosphorylation of ERK1/2 in OSCC cells treated with VitD was significantly inhibited, while total ERK1/2 was not changed (Figure 5A). Therefore, we further confirmed that MAPK was an important regulator of VitD. Similarly, we tested whether LUCAT1 also affects the MAPK signalling pathway. By exploring possible regulatory mechanisms downstream of LUCAT1 on MAPK, we found that MAPK phos- phorylation was significantly inhibited and affected the activation of the MAPK signalling pathway in the si-LUCAT1 group and shLUCAT group (Figure 5B).

In conclusion, LUCAT1 was a key molecule responsible for proliferation of OSCC. Vitamin D inhibited MAPK signalling pathway activation by suppressing LUCAT1, which suppressed the proliferation of oral cancer (Figure 6).
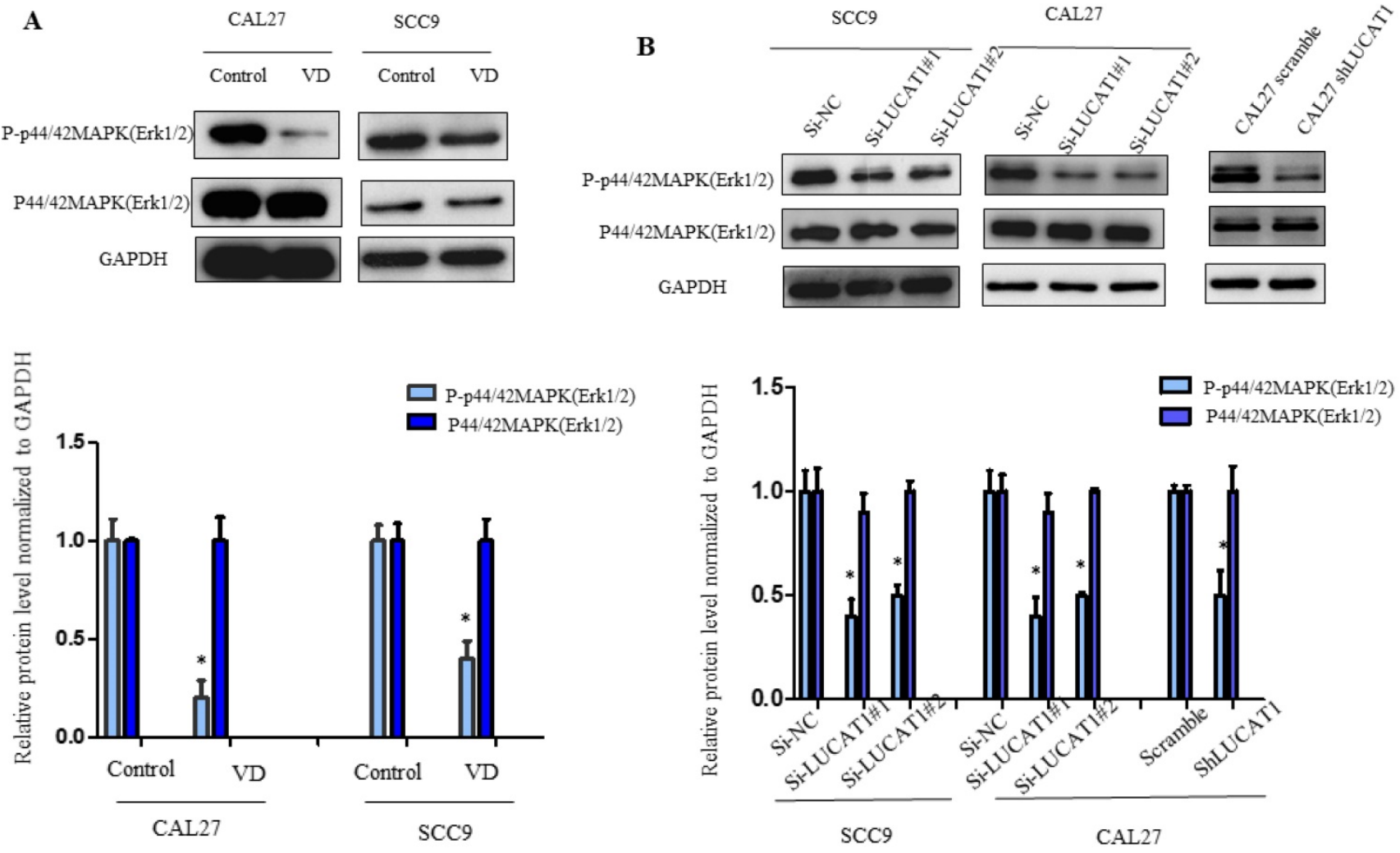

Figure 5. Effect of vitamin D and LUCAT1 on the MAPK signalling pathway. A. The protein level of the MAPK signalling pathway was examined after vitamin D treatment. B. Detection of MAPK signalling pathway protein levels after low expression of LUCAT1.

TSCC Proliferation

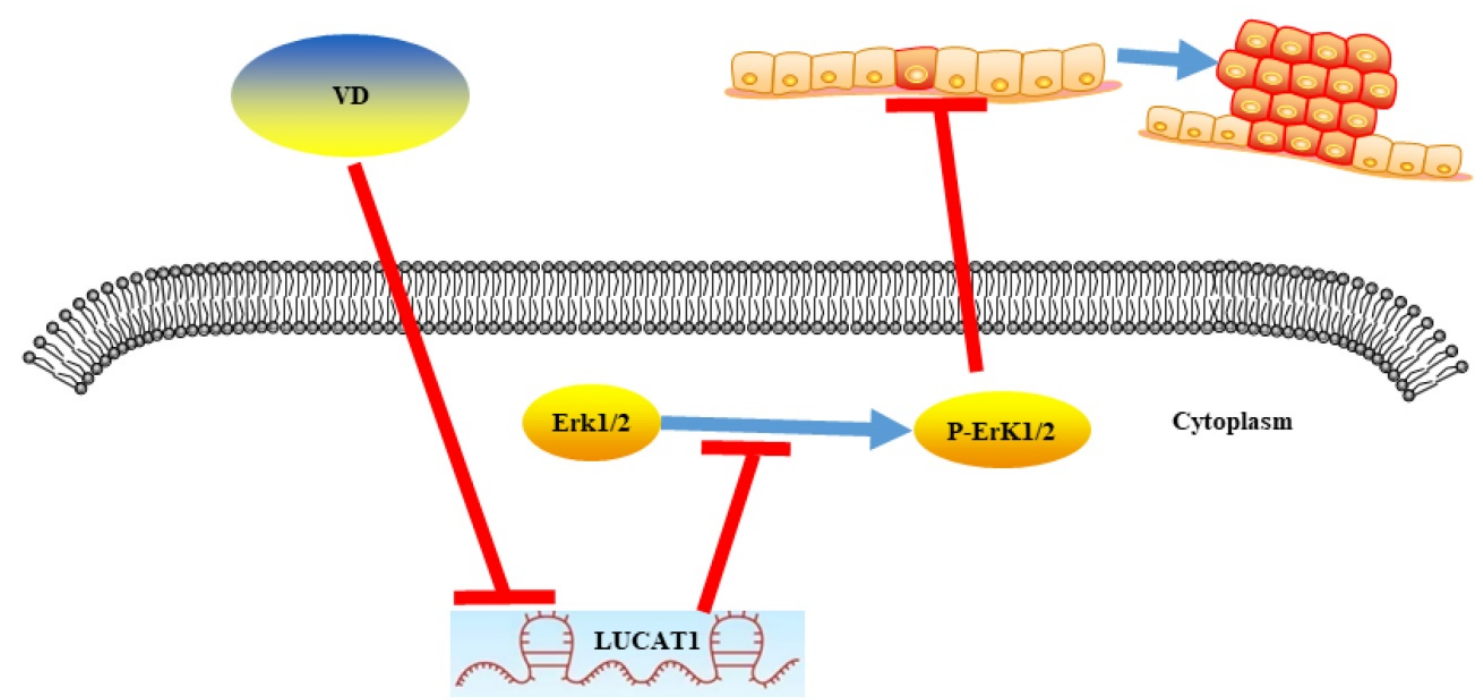

Figure 6. A schematic model showing that vitamin D inhibited the growth of OSCC cells through the LUCAT1-MAPK signalling pathway. 


\section{Discussion}

Vitamin D has been confirmed to have antitumour effects in previous studies. Evidence from epidemiologic, preclinical, and clinical studies indicates that $\mathrm{VitD}$ might have usability as a therapeutic agent in cancer patients [18]. VitD has an antitumour effect through modulation of inflammation, cell proliferation, cell differentiation, angiogenesis, invasive and metastatic potential, and apoptosis [19]. Previously, it has been reported that VitD3 and 13-cis retinoic acid have equipotent antiproliferative effects on tongue squamous cell carcinoma (SCC-25) cells [20]. Additionally, $1,25(\mathrm{OH}) 2 \mathrm{D} 3$ has been shown to inhibit the growth of HNSCC cells through the upregulation of cell cycle inhibitor p18 expression [21]. We previously found that VitD promotes the cisplatin sensitivity of oral squamous cell carcinoma by inhibiting LCN2modulated NF-kB pathway activation through RPS3 [11]. This study further demonstrated that VitD can significantly inhibit the proliferation of OSCC; therefore, VitD may be used as a drug to prevent and treat oral cancer in clinical practice.

Recently, efforts have been dedicated to elucidating the biological functions and clinical impact of long noncoding RNAs, and studies have revealed that lncRNAs might be novel biomarkers for several types of human cancers, including OSCC [22]. It has been reported that some lncRNAs play an important role in the development of OSCC [23, 24]. In this study, we determined that VitD inhibited OSCC cell proliferation through the regulation of LUCAT1. We examined the expression levels of LUCAT1 in OSCC clinical specimens and cell lines and found that LUCAT1 was significantly increased in OSCC samples. High LUCAT1 expression was an independent prognostic factor for liver cancer [25]. Therefore, LUCAT1 can be used as a biological indicator and a prognostic factor of OSCC, and LUCAT1 is a clinical marker that can be used for patient management plans to improve treatment.

Esma Karkeni et al. demonstrated for the first time that VitD modulates the expression of miRNAs in adipocytes in vitro and in adipose tissue in vivo through its impact on the NF-kB signalling pathway [26]. We investigated the impact of VitD on lncRNA expression in OSCC using high-throughput sequencing, and we identified for the first time lncRNA LUCAT1 as a potential VitD target. How LUCAT1 is activated directly by VitD should be further explored.

Lung cancer-associated transcript 1 (LUCAT1) was first reported to be involved in smoking-related lung cancer. It has been reported that LUCAT1 has an antitumour effect by modulating miRNA expression and signalling pathways in clear cell renal cell carcinoma, bladder cancer, colorectal cancer, hepatocellular carcinoma, breast cancer and cervical cancer [27].

To identify molecular mechanisms regulating this process, several approaches were combined. Previous studies have shown that LUCAT1 promoted proliferation and invasion in clear cell renal cell carcinoma cells through the AKT/GSK-3 $\beta$ signalling pathway [28]. To the best of our knowledge, the mitogen-activated protein kinase/extracellular signalregulated kinase (MAPK/ERK) pathway has been reported to be associated with cell proliferation, differentiation, migration, senescence and apoptosis. Therefore, a western blot experiment was performed showing that low expression of LUCAT1 can significantly inhibit the activation of the mitogenactivated protein kinase/extracellular signalregulated kinase (MAPK/ERK) pathway, thus affecting the proliferation of OSCC. Based on our results, it is reasonable to conclude that LUCAT1 inhibits the MAPK signalling pathway in tongue squamous cell carcinoma.

Despite advances in surgical techniques and postoperative radiotherapy, the overall 5-year survival of OSCC patients has not significantly improved in the last 50 years. Therefore, new therapies for OSCC are urgently needed. To find a more effective treatment, in addition to early surgeryradiotherapy and chemotherapy sequence treatment, EGFR inhibitors and COX-2 inhibitors photodynamic therapy, as well as FOXM1 and PD-L1 inhibitors, have been proposed [29]. However, all have led to nonspecific cell death. VitD can reduce the expression of the tumour-promoting factor LUCAT1 and inhibit the proliferation of OSCC. VitD supplementation should be a better choice than using LUCAT1 inhibitors to inhibit the proliferation and progression of OSCC. Further clinical trials are certainly needed for the dosage and time-course effects.

\section{Conclusion}

In summary, VitD treatment significantly inhibited the expression of LUCAT1, downregulated MAPK activation, and inhibited OSCC growth. These results reveal the therapeutic potential of VitD for OSCC treatment, prevention and delay of the onset of recurrence. Thus, improvement of VitD status with sensible sun exposure, VitD supplementation and ingesting foods containing VitD is a reasonable strategy to reduce the risk of malignancy. In addition, LUCAT1 is expected to be a new target for biotherapy. 


\section{Abbreviations}

VitD: Vitamin D; OSCC: Oral squamous cell carcinoma; TSCC: Tongue squamous cell carcinoma; lncRNAs: Long noncoding RNAs; MAPK: Activation of the mitogen protein kinase; LUCAT1: Lung cancer associated transcript; RT-qPCR: Quantitative real time polymerase chain reaction; SiRNA: Small interfering RNA.

\section{Supplementary Material}

VitD-regulated lncRNAs.

http://www.jcancer.org/v11p5971s1.xlsx

\section{Acknowledgements}

This work was supported by grants from Guangdong Provincial Key Laboratory of Malignant Tumor Epigenetics and Gene Regulation, Sun Yat-Sen Memorial Hospital, Sun Yat-Sen University, from Guangdong Science and Technology Department (2017B030314026), National Natural Science Foundation of China (\#81772892), the Guangdong Province Natural Science Foundation (2016A030313348, 2019A1515011932), Science and Technology Program of Guangdong (\#2019A141405062).

\section{Authors' contributions}

TTJ Jin, $Z Q H$ and $Y Z$ conceived and designed the study. TTJ, YG, ZXH, QYZ, and ZSH performed the experiments. TTJ and $Y G$ wrote the paper. $Z Q H$, $Y Z$ and $Y G$ reviewed and edited the manuscript. All authors read and approved the manuscript.

\section{Ethical approval}

This research was conducted in accordance with international guidelines and the ethical standards outlined in the Declaration of Helsinki. This study was approved by the Sun Yat-sen Memorial Hospital Institutional Review Board. All animal experiments were conducted following the Ministry of Health national guidelines for housing and care of laboratory animals and performed in accordance with institutional regulations after review and approval by the Institutional Animal Care and Use Committee at Sun Yat-sen University.

\section{Availability of data and materials}

The datasets used for the current study are available from the corresponding author on reasonable request. All data generated or analysed during this study are included in this published article.

\section{Competing Interests}

The authors have declared that no competing interest exists.

\section{References}

1. Siegel RL, Miller KD, Jemal A. Cancer statistics, 2019. CA Cancer J Clin. 2019;69(1):7-34

2. D'Alessandro AF, Pinto FR, Lin CS, et al.. Oral cavity squamous cell carcinoma: factors related to occult lymph node metastasis. Braz J Otorhinolaryngol. 2015;81(3):248-254.

3. Leemans CR, Braakhuis BJ, Brakenhoff RH. The molecular biology of head and neck cancer. Nat Rev Cancer. 2011;11(1):9-22.

4. Parikh P, Patil V, Agarwal JP, et al.. Guidelines for treatment of recurrent or metastatic head and neck cancer. Indian J Cancer. 2014;51(2):89-94.

5. Jiang YY, Lin DC, Mayakonda A, et al.. Targeting super-enhancer-associated oncogenes in oesophageal squamous cell carcinoma. Gut. 2017;66(8):13581368.

6. Ma Y, Yu WD, Hidalgo AA, et al.. Inecalcitol, an analog of 1,25D3, displays enhanced antitumor activity through the induction of apoptosis in a squamous cell carcinoma model system. Cell Cycle. 2013;12(5):743-752.

7. Lee J, Park SH. Tumor-suppressive activity of 1,25-dihydroxyvitamin D3 against kidney cancer cells via up-regulation of FOXO3. Biosci Biotechnol Biochem. 2016;80(10):1947-1953.

8. Xie SP, Pirianov G, Colston KW. Vitamin D analogues suppress IGF-I signalling and promote apoptosis in breast cancer cells. Eur J Cancer. 1999;35(12):1717-1723.

9. Huang Z, Liu Y, Huang Z, et al.. 1,25-Dihydroxy vitamin D3 alleviates salivary adenoid cystic carcinoma progression by suppressing GPX1 expression through the NF-kB pathway. Int J Oncol. 2016;48(3):1271-1279.

10. Gan X, Chen B, Shen Z, et al.. High GPX1 expression promotes esophageal squamous cell carcinoma invasion, migration, proliferation and cisplatinresistance but can be reduced by vitamin D. Int J Clin Exp Med. 2014;7(9):2530-2540.

11. Huang Z, Zhang $\mathrm{Y}$, Li H, et al.. Vitamin D promotes the cisplatin sensitivity of oral squamous cell carcinoma by inhibiting LCN2-modulated NF-kB pathway activation through RPS3. Cell Death Dis. 2019;10(12):936.

12. Djebali S, Davis CA, Merkel A, et al.. Landscape of transcription in human cells. Nature. 2012;489(7414):101-108.

13. Iyer MK, Niknafs YS, Malik R, et al.. The landscape of long noncoding RNAs in the human transcriptome. Nat Genet. 2015;47(3):199-208.

14. Dai MY, Zhang Y, Zhang ZN, et al.. Effects of long non-coding RNA HOTAIR on proliferation, migration and invasion of tongue squamous cell carcinoma. Oral Sci Res. 2018;34:627-631.

15. Liang J, Liang L, Ouyang K, et al.. MALAT1 induces tongue cancer cells' EMT and inhibits apoptosis through Wnt/beta-catenin signaling pathway. J Oral Pathol Med. 2017;46:98-105.

16. Saad El-Din S, Rashed L, Medhat E, et al.. Active Form of Vitamin D Analogue Mitigates Neurodegenerative Changes in Alzheimer's Disease in Rats by Targeting Keap1/Nrf2 and MAPK-38p/ERK signaling pathways [published online ahead of print, 2020 Jan 23]. Steroids. 2020;108586.

17. Gan X, Chen B, Shen Z, et al.. High GPX1 T-type Ca2+ channel inhibition induces p53-dependent cell growth arrest and apoptosis through activation of p38-MAPK in colon cancer cells expression promotes esophageal squamous cell carcinoma invasion, migration, proliferation and cisplatin-resistance but can be reduced by vitamin D. Int J Clin Exp Med. 2014;7(9):2530-2540.

18. Fedirko V, Bostick RM, Flanders WD, et al.. Effects of vitamin d and calcium on proliferation and differentiation in normal colon mucosa: a randomized clinical trial. Cancer Epidemiol Biomarkers Prev. 2009;18(11):2933-2941.

19. Bandera Merchan B, Morcillo S, Martin-Nuñez G, et al.. The role of vitamin D and VDR in carcinogenesis: Through epidemiology and basic sciences. J Steroid Biochem Mol Biol. 2017;167:203-218.

20. Enepekides DJ, Black MJ, White JH. The independent and combined effects of RAR-, RXR-, and VDR-selective ligands on the growth of squamous cell carcinoma in vitro. J Otolaryngol. 1999;28(2):83-89.

21. Gedlicka C, Hager G, Weissenböck M, et al.. 1,25(OH)2Vitamin D3 induces elevated expression of the cell cycle inhibitor p18 in a squamous cell carcinoma cell line of the head and neck. J Oral Pathol Med. 2006;35(8):472478.

22. Lee JT. Epigenetic regulation by long noncoding RNAs. Science. 2012; 338(6113):1435-1439.

23. Shao TR, Zheng ZN, Chen YC, et al.. LncRNA AC007271.3 promotes cell proliferation, invasion, migration and inhibits cell apoptosis of OSCC via the Wnt/ $\beta$-catenin signaling pathway. Life Sci. 2019;239:117087.

24. Liu M, Gao X, Liu CL. Increased expression of IncRNA FTH1P3 promotes oral squamous cell carcinoma cells migration and invasion by enhancing PI3K/ Akt/GSK3b/Wnt/ $\beta$-catenin signaling. Eur Rev Med Pharmacol Sci. 2018; 22(23):8306-8314.

25. Jiao $Y$, Li Y, Ji B, et al.. Clinical Value of lncRNA LUCAT1 Expression in Liver Cancer and its Potential Pathways. J Gastrointestin Liver Dis. 2019;28(4):439447. Published 2019 Dec 9.

26. Karkeni E, Bonnet L, Marcotorchino J, et al. Vitamin D limits inflammationlinked microRNA expression in adipocytes in vitro and in vivo: A new mechanism for the regulation of inflammation by vitamin D. Epigenetics. 2018;13(2):156-162.

27. Lou Y, Yu Y, Xu X, et al.. Long non-coding RNA LUCAT1 promotes tumourigenesis by inhibiting ANXA2 phosphorylation in hepatocellular carcinoma. J Cell Mol Med. 2019;23(3):1873-1884. 
28. Zheng Z, Zhao F, Zhu D, et al.. Long Non-Coding RNA LUCAT1 Promotes Proliferation and Invasion in Clear Cell Renal Cell Carcinoma Through AKT/ GSK-3 $\beta$ Signaling Pathway. Cell Physiol Biochem. 2018;48(3):891-904.

29. Wang W, Tan J. Co-inhibition of BET proteins and PD-L1 as a potential therapy for OSCC through synergistic inhibition of FOXM1 and PD-L1 expressions. J Oral Pathol Med. 2019;48(9):817-825. 